\title{
Challenges in nanoscale physics of wetting phenomena
}

\author{
S. Afkhami ${ }^{1, a}$, T. Gambaryan-Roisman ${ }^{2, b}$, and L.M. Pismen ${ }^{3, c}$ \\ ${ }^{1}$ Department of Mathematical Sciences, New Jersey Institute of Technology, Newark, \\ NJ, USA \\ ${ }^{2}$ Institute for Technical Thermodynamics, Technische Universität Darmstadt, \\ Darmstadt, Germany \\ ${ }^{3}$ Department of Chemical Engineering, Technion - Israel Institute of Technology, \\ Haifa, Israel
}

Received 23 July 2020 / Accepted 23 July 2020

Published online 14 September 2020

Abstract. We describe the aims and content of this issue.

\section{Introduction}

Within the broad range of flow phenomena, problems involving fluid interfaces, and their interaction with solid boundaries, are one of the most interesting and challenging areas. Topics related to wetting phenomena and their applications attract continuing interest in the scientific community, in view of their relevance to micro- and nanofluidic applications, ink-jet printing, nanotechnology, prevention of ice accretion, heat transfer enhancement, etc. Further progress in development of these technologies is impossible without detailed understanding of nanoscale mechanisms governing wetting phenomena, both in conventional wetting and in wetting of complex liquids on complex solids. The increasing activity of scientific community is reflected in a number of emerging focused collaborative research initiatives on the national, European, and international level. These collaborative projects are devoted both to elucidating fundamental wetting mechanisms and their interaction with transport of mass, momentum and energy, and to controlling wetting phenomena in applications-relevant systems involving complex fluids (surfactant solutions, suspensions, emulsions) and complex surfaces.

This special issue nucleated at the workshop "Challenges in nanoscale physics of wetting phenomena" (NaWet) that took place at the Max Planck Institute for the Physics of Complex Systems in Dresden in August 2019, but is in no way identical to the workshop program. It aims at addressing recent developments and open questions related to computational, analytical, and experimental aspects of physics of wetting phenomena. A continuing attention to this theme had already inspired a Discussion and Debate issue of EPJ ST [1].

In many natural phenomena and practical applications, the wetted substrate is not smooth; this is a case for wetting of rough substrates, of structured substrates or

\footnotetext{
a e-mail: shahriar.afkhami@njit.edu

b e-mail: gtatiana@ttd.tu-darmstadt.de

c e-mail: pismen@technion.ac.il
} 
structures coated with porous coatings, as well as spontaneous or forced wetting of porous materials; nanoscale interactions influence even wetting of smooth surfaces. This special issue contains theoretical and experimental works covering a wide range of topics, and highlights the challenge of bridging widely separated scales, from macroscopic to molecular, that bears upon these phenomena. We hope that this will pave the way toward future developments in this scientifically challenging and practically important problem.

In the following, we briefly annotate the contributions to the issue, separating those emphasizing macroscopic or multiscale approach, and ending with the discussion contributions. The papers are listed in the order of submission within each section.

\section{Macroscopic experiments and modeling}

Ludwicki and Steen [2] present a study of sweeping of wetted area by the sessile drop coalescence on a solid substrate. The observations illustrate the influence of the solid's wettability on the sweeping efficiency, and are complemented by volume-offluid simulations.

The experiments by Seiler et al. [3] illustrate the interaction between an airdriven, wall-bound drop, and a groove in the wall of a channel, leading to three alternative outcomes: the drop passing over the groove, or being fully captured, or being captured after first wetting the rear side of the groove. Threshold conditions for different outcomes are predicted by a model based on scaling arguments.

Kim et al. [4] investigate with the help of tomographic particle image velocimetry the internal flow pattern induced by an evaporating droplet with a stick-and-slip motion of the contact line. They detect the formation of an azimuthal vortex pair and explain this phenomenon by a theoretical model taking into account the relation between the contact line motion and the evaporating flux.

O'Brien et al. [5] apply the volume-of-fluid method to a detailed pore-scale numerical simulation of a two-phase flow in a porous medium. An ordered porous medium is modeled using the immersed boundary method. The displacement patterns are shown to be affected by both the capillary number and wetting characteristics that govern the transition between stable front propagation and the viscous fingering regimes.

Ghillani et al. [6] model the mechanisms of imbibition in porous coatings by the simultaneous liquid capillary rise and evaporation within a corner between a substrate and a fiber. Their computations based on a finite elements method and compared to experimental data, demonstrate that imbibition is enhanced by the capillary rise in the corner between the substrate and the first layer of nanofibers, and is depressed when evaporation becomes significant.

\section{Bridging the scales}

Thiele and Hartmann [7] have developed a gradient dynamics model of the droplet spreading on polymer brushes based on a thin film approximation. The wetting dynamics is coupled with the dynamics of swelling, absorption, and diffusion. They highlight a remarkable difference between the macroscopic contact angle defined by extrapolating the drop's shape, and the mesoscopic contact angle defined at the inflection point of the drop profile.

Limat [8] reconsiders the problem of a sliding drop that develops a conical tail at the rear, taking into account friction of a microscopic origin at the contact line. In 
addition, he proposes a simple model to describe the evaporation of liquid. He also emphasizes the key role of evaporation in the microscale dynamics.

Fricke and Bothe [9] address the issue of relieving the moving contact line singularity from the point of view of compatibility analysis. They analyze two continuum mechanic models: the standard Navier slip model and the model in which the interfacial layer is considered separately from the fluid bulk.

Herbaut et al. [10] develop a model predicting the pinning condition of the contact line on a substrate at temperatures below the freezing temperature of the wetting liquid. The most important model predictions include the dependence of the apparent contact angle on the degree of undercooling and the critical velocity for the transition to stick-slip motion. A limitation of the model is treating the microscopic scale as an adjustable parameter.

Alizadeh-Birjandi et al. [11] study experimentally freezing on feathers taken from both cold-weather and warm-weather penguins, supporting the hypothesis that the origin of anti-icing properties of cold-weather penguin's feathers comes from a unique combination of their macroscopic structure, the nanoscale topography of its barbules, and the hydrophobicity of its preen oil.

Lācis et al. [12] develop a molecular dynamics framework for an atomistic water model to simulate a nanoscopic drop between two moving plates, taking into account hydrogen bonding between the water molecules and the solid substrate that leads to a sub-molecular slip length at the contact line. Comparison of their results with standard continuum computations demonstrate the importance of the molecular-scale mobility and local slip for accurate modeling of the moving contact line.

Fullana et al. [13] model dynamic wetting in the curtain coating configuration, using a volume-of-fluid method with the Navier slip boundary condition to regularize the solution at the contact line. Their results predict the onset of wetting failure but a comparison with the available literature suggests Navier slip coupled with a constant contact angle might be insufficient to regularize the solution at the contact line.

Zigelman et al. [14] study the influence of the ionic strength on particles deposition patterns from suspensions, and explain its strength by the growth of local concentration of both particles and ions due to microscale convection and enhanced evaporation near the contact line. The process is sensitive to the ion strength that reduces the energy barrier to deposition by screening of electrostatic forces.

\section{Discussion}

In the only Discussion contribution to the present issue, Shikhmurzaev [15] presents a detailed review of mathematical models of dynamic wetting. He challenges the common view shared by a number of models that the dynamic contact angle is a unique function of a contact line speed. His "litmus tests" for mathematical models are criticized by Bothe [16], and the vivid discussion is continued with the rebuttal by Shikhmurzaev [17]. Shikhmurzaev's views, which bear upon the crucial question of relation between macroscopic and molecular-scale factors in wetting phenomena have been already a subject of debate in the EPJ ST issue edited by Velarde [1]. We share his opinion that the contact angle, either static or dynamic, is ill-defined: it depends on where and how it is measured. It is more controversial that, in the spirit of classical fluid dynamics, he insists that molecular-scale interactions cannot enter macroscopic equations but should be introduced via boundary conditions. It is well known that classical hydrodynamic equations with classical boundary conditions fail to resolve the motion of a contact line. Standard equations work perfectly in the bulk, but boundary conditions have to take into account molecular interactions. Analytical techniques (e.g., [18]) derive boundary conditions for macroscopic flow 
through multiscale analysis matching molecular and macroscopic scales, but their applications are limited. Such matching can be, in principle, implemented with the help of diffuse interface models and/or molecular dynamics simulations, but we are still waiting for the emergence of such a kind of multiscale numerical modeling.

Open access funding provided by Projekt DEAL.

Open Access This is an open access article distributed under the terms of the Creative Commons Attribution License (http://creativecommons.org/licenses/by/4.0), which permits unrestricted use, distribution, and reproduction in any medium, provided the original work is properly cited.

Publisher's Note The EPJ Publishers remain neutral with regard to jurisdictional claims in published maps and institutional affiliations.

\section{References}

1. M.G. Velarde, Eur. Phys. J. Special Topics 197, 1 (2011)

2. J.M. Ludwicki, P.H. Steen, Eur. Phys. J. Special Topics 229, 1739 (2020)

3. P.M. Seiler, I.V. Roisman, C. Tropea, Eur. Phys. J. Special Topics 229, 1757 (2020)

4. H. Kim, N. Belmiloud, P.W. Mertens, Eur. Phys. J. Special Topics 229, 1771 (2020)

5. A. O'Brien, S. Afkhami, M. Bussmann, Eur. Phys. J. Special Topics 229, 1785 (2020)

6. N. Ghillani, M. Heinz, T. Gambaryan-Roisman, Eur. Phys. J. Special Topics 229, 1799 (2020)

7. U. Thiele, S. Hartmann, Eur. Phys. J. Special Topics 229, 1819 (2020)

8. L. Limat, Eur. Phys. J. Special Topics 229, 1833 (2020)

9. M. Fricke, D. Bothe, Eur. Phys. J. Special Topics 229, 1849 (2020)

10. R. Herbaut, J. Dervaux, P. Brunet, L. Royon, L. Limat, Eur. Phys. J. Special Topics 229, 1867 (2020)

11. E. Alizadeh-Birjandi, F. Tavakoli-Dastjerdi, J. St. Leger, K.F. Faull, S.H. Davis, J.P. Rothstein, H.P. Kavehpour, Eur. Phys. J. Special Topics 229, 1881 (2020)

12. U. Lācis, P. Johansson, T. Fullana, B. Hess, G. Amberg, S. Bagheri, S. Zaleski, Eur. Phys. J. Special Topics 229, 1897 (2020)

13. T. Fullana, S. Zaleski, S. Popinet, Eur. Phys. J. Special Topics 229, 1923 (2020)

14. A. Zigelman, E. Homede, O. Manor, Eur. Phys. J. Special Topics 229, 1935 (2020)

15. Y.D. Shikhmurzaev, Eur. Phys. J. Special Topics 229, 1945 (2020)

16. D. Bothe, Eur. Phys. J. Special Topics 229, 1979 (2020)

17. Y.D. Shikhmurzaev, Eur. Phys. J. Special Topics 229, 1989 (2020)

18. L.M. Pismen, Y. Pomeau, Phys. Fluids 16, 2604 (2004) 